\title{
Sources of pre-admission medication information: Observational study of the accuracy and availability.
}

Fitzsimons M, Grimes T, Galvin M.

This is the peer reviewed version of the following article: [Sources of preadmission medication information: Observational study of the accuracy and availability], which has been published in final form at [http://onlinelibrary.wiley.com/doi/10.1111/j.2042-7174.2011.00154.x/abstract DOI 10.1111/j.2042-7174.2011.00154.x]. This article may be used for noncommercial purposes in accordance with Wiley Terms and Conditions for Self-Archiving.

Article first published online: 19 AUG 2011

Fitzsimons M, Grimes T, Galvin M. Sources of pre-admission medication information: Observational study of the accuracy and availability. International Journal of Pharmacy Practice (2011);19(6):408-416. 


\section{Introduction}

Medication history errors at hospital admission have been identified in up to $67 \%$ of cases.[1] Medicines reconciliation is defined by the Institute for Healthcare Improvement $(\mathrm{IHI})$ in the US as the process of creating the most accurate list possible of all medications a patient is taking - including drug name, dosage, frequency, and route - and comparing that list against the physician's admission, transfer, and/or discharge orders, with the goal of providing correct medications to the patient at all transition points within the hospital.[2] There is international evidence that failure to reconcile medications at hospital admission can result in interruption of drug therapy and the inappropriate use of medication. This may result in an adverse drug reaction or deterioration of a chronic condition with the potential for prolonged hospital stay or unplanned readmission.[3-7] The IHI and the National Institute for Health and Clinical Excellence (NICE) together with the National Patient Safety Agency (NPSA) in the UK issued guidance on the implementation of medicines reconciliation processes at the interfaces of care.[2, 8] In the Republic of Ireland, the Report of the Commission on Patient Safety and Quality Assurance, Building a Culture of Patient Safety, (2008) recommended that medication reconciliation be undertaken at all points of transfer of care.[9] Undertaking reconciliation is resource intensive and time consuming and to ensure efficiency clinician access to reliable pre-admission medication (PAM) information is important.[10]

The concept of a "gold standard" pre-admission medication list (GS-PAML) was introduced in the US by Pippins and colleagues, 2008, as the list resultant from undertaking a comprehensive medication history, using all available sources of information, to generate the most complete and accurate list of the medications the patient was actually using prior to hospital admission.[5] Ideally, pre-admission medication lists (PAMLs) should not merely list the medications prescribed or dispensed to the patient, but reflect what the patient was actually using prior to admission, taking into account any non-compliance and non-prescribed medications.[11] There is evidence from the UK, Denmark and the Netherlands that a variety of sources should be used to obtain a reliable and accurate PAML.[11-15] Each individual source has its limitations.[15] For example, patients may not report non-compliance; being acutely unwell on admission to hospital may impact on recall; and acute admissions by their nature are unplanned, so patients may not bring in 
their own medications from home. Community pharmacy records usually capture data regarding prescription-only medications (POM) dispensed but not items purchased over the counter (OTC). Patients may obtain medication from more than one community pharmacy. Similarly, general practice (GP) records may not include OTC medications, nor items prescribed by other prescribers, such as hospital physicians.[15] Patient non-adherence to prescribed medications is a significant problem world wide[16] and may also lead to inaccuracies in lists provided by the GP and community pharmacy. The internationally recognised challenge of communicating complete and accurate medication information between primary and secondary care settings adds complexity to elucidating the patient's actual PAML.[13, $15,17-19]$

Research conducted in Northern Ireland identified that all sources of medication history surveyed had low levels of accuracy.[20] When compared to the accurate drug history produced by the clinical pharmacist, $25 \%$ of GP histories, $22 \%$ of patient/carer histories, $18 \%$ of community pharmacy histories and $18 \%$ of patient own drugs (PODs) histories had zero discrepancies $(n=371)$. Other UK studies have found GP letters to be an inaccurate source.[3, 21] Dutch research has shown that the use of community pharmacy records in conjunction with patient involvement is an effective method of constructing an accurate medication history.[15, 22] Little is known about the accuracy of sources of PAM information used on admission to hospital in Ireland. Furthermore, although much work has been undertaken to assess the accuracy of sources of medication history information in a number of settings, little research has reported on the accessibility or availability of sources, an important consideration in this resource limited time. This paper sought to quantify the accessibility and reliability of sources of medication history data and to categorise the types of disagreements between the lists provided by each source and the GSPAML.

\section{Method}

\section{Study setting and participants}

The study was undertaken across two acute hospitals operating 24 hour accident and emergency (A\&E) departments: The Adelaide and Meath Hospital Dublin, incorporating the National Children's Hospital (AMNCH) is a 600 bed academic teaching hospital that serves a large urban population in south west Dublin; Naas General Hospital (NGH) serves the more rural catchment area of Kildare and west 
Wicklow and has 243 beds. Adult patients using at least three regular medicines and admitted via the $A \& E$ department were eligible for inclusion. For the purpose of the study, patient use of medication included medication prescribed, dispensed or used. Patients were excluded if they were: transferred from another acute hospital; unavailable at the time of data collection (e.g. in theatre); isolated for infection control reasons; non English speaking and no interpreter available; and admitted more than once during the study period. The research proposal was reviewed and approved by the Ethics Committees in each hospital. In adherence with the decision of the NGH Ethics Committee, NGH patients for whom the researcher was unable to obtain verbal consent from the patient or their carer and those who presented with a psychiatric complaint were also excluded. Study participants were randomly selected on a daily basis from those who fulfilled the inclusion criteria.

\section{Research design and data collection}

This was an observational study. Data collection was carried out Monday to Friday during normal working hours in March and April 2009. Throughout the study the research team met on a regular basis to ensure consistency in the approach to data collection and coding. Data were collected within 24 hours of presentation to A\&E (72 hours for weekend admissions).[8] Demographic and clinical data were recorded from the healthcare record including: age; gender; referral source; whether the patient was transferred to hospital by ambulance; number of co-morbidities and medications. For each patient, a comprehensive medication history was undertaken and a GS-PAML was compiled. This was achieved by interviewing the patient or their carer to elucidate their account of the PAML, collecting a PAML from each of the sources listed below and then providing the patient/carer an opportunity to clarify any disagreements identified between the lists and their initial list, thus identifying their actual medication use:

- Patient's own drugs (PODs),

- GP referral letter,

- GP staff personal communication,

- Community pharmacy personal communication,

- Previous inpatient Kardex, if less than six months old,

- Previous inpatient discharge summary, if less than six months old,

- Nursing home (NH) lists and

- NH staff personal communication. 
Fitzsimons, Grimes, Galvin.

One quarter of the Irish adult population have been identified as having the lowest level of literacy[23]. In order to maximise the patient's involvement in the interview, a list of possible interview questions was developed, submitted to and amended by the National Adult Literacy Agency (NALA) to ensure they were phrased in "plain English" (Appendix 1). Whilst interviewing the patient, the researchers observed and recorded whether the patient used an aide de memoir (e.g. handwritten list) in recalling their PAML.

\section{Outcome measures}

From each PAM source, data were collected regarding the frequency of:

A - Potential for use

A dichotomous variable that reflected whether each source of medication history data had the potential to be used or not. For example, if a patient lived at home, there was no potential to use a nursing home as a source of information.

B - Availability at time of undertaking history

The healthcare record was examined to locate and record the availability of any GP referral letters, nursing home correspondence, past (within last six months) inpatient Kardexes and discharge summaries. PODs were deemed available if the patient brought with them to hospital any of their own medications. For personal communication (GP surgery, community pharmacy, nursing home) the researcher attempted to make contact by telephone on three separate occasions over at a thirty minute period. If contact was not made the source was recorded as unavailable.

C - Complete agreement of medication details between PAML and GS-PAML Defined as agreement between PAML and GS-PAML of the drug name, dose, frequency, route and formulation for all medications on the GS-PAML. Nonagreement was recorded and categorised for each individual medication.

D - Agreement of allergy status between PAML and GS-PAML.

Allergy agreement was defined as agreement with one of the following:

- substance or drug name(s) where the patient had a hypersensitivity or

- documentation/ communication that there was no known drug allergy. 
Fitzsimons, Grimes, Galvin.

\section{Data analysis}

Based on the findings of a previous survey, equal weighting was apportioned to B, C and D.[24] One outcome measure, encompassing reliability and availability, was identified by applying the formula $\mathbf{A}^{\star}(\mathbf{B}+\mathbf{C}+\mathbf{D})$.

In order to ensure consistency between researchers in constructing the GS-PAML, inter-rater reliability was assessed on a random selection of patients. Each researcher independently compiled the GS-PAML for five patients from each study site (total 10). Kappa statistics ( $\mathrm{K}$ ) were generated to test if the frequency of agreement exceeded that expected by chance.

Data were inputted into SPSS version 16 for support in analysis, assigning a case to each medication surveyed. Once complete the data were aggregated to provide summary data for each patient. Following data input a selection of range and consistency checks were applied to remove any obvious errors. Associations between categorical variables were investigated using the chi-square test, whilst correlations between continuous variables were investigated using Spearman's test. Statistical significance was set as $P<0.05$.

\section{Results}

\section{Study population}

A total of 134 patients were recruited to the study. The median age was 71 years (range 18-92), and there was a median of three co-morbidities (range one to 10) and nine concomitant medications (range 3-22) per patient. Almost half (46\%) were female and the majority (84\%) were admitted for medical rather than surgical care. Most $(92.5 \%)$ patients attended the same community pharmacy "all of the time" to obtain their prescription medication. Over a third (35\%) of patients used an aide de memoir in recalling their PAML and greater than one third (34\%) used at least one OTC medication. An additional 221 patients were screened but excluded, the majority (64\%) because they used less than three regular medications.

A total of 1431 medications were surveyed. Using the British National Formulary (BNF) categorisation, the most frequent types surveyed were: cardiovascular (36.1\%), central nervous system (18.1\%), gastrointestinal $(12.1 \%)$, respiratory $(8.7 \%)$ and endocrine $(7.5 \%)$. The majority (95\%) of PAMs were POMs and the remainder $(5 \%)$ were OTC drugs that were obtained in the community without a prescription. 


\section{Inter-rater reliability}

There was complete agreement in medication name, dose, frequency, formulation and route between the two researchers for 99 of the 110 medications assessed (90\%), implying moderate agreement (Kappa 0.52).[25]

\section{A. Potential to use the PAM source}

Personal communication with the community pharmacy and GP staff and PODs had the potential to be used for all patients surveyed (Table 1). Two fifths (40\%) of patients were referred to A\&E by a GP, thereby providing potential to use a GP referral letter. Greater than one third (37\%) of patients were inpatients in the study hospital in the previous six months, providing potential to use a previous inpatient Kardex and discharge summary and $7 \%$ were $\mathrm{NH}$ residents.

\section{B. Availability of potential sources}

$\mathrm{NH}$ staff and the community pharmacy were identified as the most available sources (Table 1). PODs and past discharge summaries were the least available, either because patients did not bring PODs to hospital or because there was not a copy of the summary filed in the healthcare record.

\section{Agreement between PAM list and GS-PAML}

With the exception of $\mathrm{NH}$ staff, complete agreement between individual lists and the GS-PAML was low ( $\leq 17 \%$ with zero disagreements): the list from the community pharmacy agreed most frequently, followed by that from the GP staff. Analysed per medication, the community pharmacy details agreed with the GS-PAML most often (77\%), whilst the GP referral letter agreed least often (44\%). The mean number of disagreements per patient was highest for the discharge summary, GP referral, previous inpatient Kardex and POD lists (Table 1).

\section{Accuracy of allergy status}

$\mathrm{NH}$ and GP staff were identified as the most accurate sources of information regarding allergy status (Table 1 ). Information regarding allergy status was absent from the majority of GP referrals and discharge summaries surveyed. 
Fitzsimons, Grimes, Galvin.

\section{Overall grading of sources}

The community pharmacy was identified as the best source, reflective of their accessibility and accuracy, followed by the GP staff and then the previous inpatient Kardex (Table 1).

\section{FREQUENCY AND TYPES OF DISAGREEMENT}

The most common type of disagreement for all sources, except $\mathrm{NH}$ staff, was drug omission (at least $35 \%$ of disagreements) followed by dose or frequency disagreement (at least 17\%) (Figure 1). The lists obtained from the GP staff and community pharmacy contained a considerable proportion of commissions, indicating that medicines were prescribed and dispensed which the patient did not actually use.

\section{Variables associated with disagreement}

If the patient used one or more OTC medication, agreement was statistically significantly less likely between the GS-PAML and PAMLs from the community pharmacy and the GP staff (Table 2). There was a positive, statistically significant correlation identified across all sources between the number of disagreements per list and the number of PAMs per patient (Table 3). Patients who used an aide de memoir in recalling their PAML were more likely to provide an initial list in agreement with the GS-PAML than those who did not $(65 \% \vee 21 \%, \chi 224.9$, df $1, P<0.05)$. There were too few patients admitted from nursing homes to enable analysis of the variables associated with disagreement.

\section{Discussion}

This study identified that for the majority of patients, the community pharmacy and GP staff were the most useful sources to corroborate the patient's initial list of PAMs, having consideration for the reliability and accessibility of each source. For the nine patients admitted from nursing home care, the findings suggest that contacting the nursing home to obtain a medication history is a pragmatic first step. Investigation was undertaken across two sites, using a random selection of patients, both of which support the generalisability of the findings beyond the study setting. The study population was adult patients using three or more regular medicines, and the findings may not be representative of other patient groups. The sample size of 134 patients is relatively small but compares favourably with other medication history studies. [4, 11 , 26] As the study was conducted during regular working hours, the findings are not reflective of out of hours or weekend practice. However, the medication history was obtained within 24 hours (72 hours at weekends) of admission, consistent with the 
NICE/NPSA recommended timeframe for medicines reconciliation.[8] A rigorous approach was undertaken to elucidate patients' medication use behaviours by ensuring the questions asked were Plain English and this adds to the reliability of the GS-PAML. Previous studies in the UK and Denmark investigated the frequency of agreement between sources of pre-admission information [11-13, 20, 27], however the authors are not aware of any study that considered the availability of that information. This approach was novel and generates important findings to support efficient collection of reliable PAML data in a busy clinical setting.

It was identified that PAMLs from each individual source of information rarely agreed with the GS-PAML ( $\leq 17 \%$ with no discrepancies), consistent with previous findings from Denmark and the UK.[11, 20, 27] This illustrates the complexity of identifying the patient's actual medication use and provides further evidence that medication histories should be built by discussion with the patient and corroboration with a least one other source. The prevalence of disagreements was highest in GP referrals and previous discharge summaries, suggestive that these sources should be used with caution or perhaps only when there is no alternative available. Discrepancies in medication lists in GP referrals[28] and discharge summaries[7, 17, 18] have been reported in Canada, Ireland, the UK and the Netherlands. The finding that the community pharmacy provided information most frequently in agreement with the GS-PAML was inconsistent with findings from the UK, where the GP was identified as the most accurate source.[20]

Omission of a medication that the patient was actually using was identified in this study as the most common type of disagreement across all sources of PAM information, consistent with published findings from Canada and the Netherlands.[4, $22,29]$ Patients were interviewed to determine for each identified omission whether they were currently using the medication. The frequency of omission may reflect that few healthcare providers have a complete view of the patient's medication use behaviour, and possible reasons for this are that patients may use non-prescription medication, or attend more than one prescriber or community pharmacy.[28] Commission was most frequently identified in the lists provided by the GP staff and the community pharmacy. A commission meant that medication was prescribed or dispensed, but not actually used by the patient. This situation may have arisen because medication lists were not updated or because the patient was non-adherent and this was unknown to the primary care clinician. Both of these problems have been reported internationally.[28, 30-32] These findings support the need to 
Fitzsimons, Grimes, Galvin.

interview the patient as part of the medication history taking process to identify their medication use patterns.

The nursing home and community pharmacy were identified in this study as most accessible at the time of undertaking the medication history. The authors are not aware of other studies that measured this output. GP staff were less accessible than the community pharmacy, whilst less than half of the patients surveyed brought their PODs to hospital. These findings are useful to guide the choice regarding which source(s) of information to contact first when conducting a medication history review. Patients or carers who used an aide de memoir, including PODs, in recalling their PAML were more accurate than those who did not. The use of a patient medicine bag or "green bag", whereby patients are encouraged to gather their medicines into a bag and bring them to hospital, is recommended in a number of UK NHS trusts and was recommended in the Care Quality Commission's report regarding management of medications after discharge[17]. The evidence from this study is that patients in Ireland should also be encouraged and facilitated to bring their aide de memoir to hospital and methods to achieve this should be explored.

There is evidence from Ireland and the UK that documentation of allergies and other drug sensitivities for hospital patients and care home residents is often absent or inaccurate.[26, 33, 34] However, there is a paucity of literature regarding such documentation in the GP or community pharmacy settings. One recent UK study of nursing home residents identified that the GP record contained drug sensitivity information for $73 \%$ of patients identified as having one or more sensitivity, but did not identify the frequency of documentation of no known allergy.[33] Regarding the care of patients with stroke, an Irish study identified that community pharmacists perceived the sharing of drug allergy information between GPs, community pharmacists and hospital prescribers was not common.[35] In this study, personal communication with the nursing home or the GP staff was identified as the best means of identifying allergy status. There was less frequent agreement between the GS-PAML and information provided by the community pharmacy, or documented in the hospital Kardex, discharge summary or GP referral. Patient and clinician awareness of allergy status is essential to facilitate appropriate medication use and the National Prescribing Centre in the UK includes allergies as part of the minimum data set to complete the reconciliation process effectively.[36] This finding highlights an opportunity to improve the sharing of allergy information between clinicians and across care settings in Ireland. 
Fitzsimons, Grimes, Galvin.

The study identified a positive correlation between the number of drugs the patient used before admission and the number of disagreements per PAML from all sources, a relationship previously identified by others in the Netherlands and the US.[28, 37] Achieving medication reconciliation at discharge is more complex amongst patients using higher numbers of medications and this finding adds further evidence to that.[38] The number of PAMLs should be used to guide prioritisation of patients to receive medication reconciliation services in hospitals where resources do not permit service delivery to every patient.

\section{Conclusion}

The community pharmacy and the GP staff were identified as the best sources of pre-admission medication information for the majority of patients, whilst communication with the nursing home staff was best for nursing home patients. Interviewing the patient was identified as a necessary part of the process to elucidate actual medication use. At least two sources should be consulted to construct the best possible pre-admission medication list. The sharing of allergy information between clinicians and across settings and the recording of this information in discharge summaries and GP referrals requires improvement. Whilst this paper identifies the sources of information which should be used in the Irish setting, it also demonstrates that use of more than two sources of information to develop a reliable PAML is advisable in all countries and that local research should be undertaken in individual countries to assess the reliability and availability of sources of PAM.

\section{Acknowledgements and Funding}

This research received no specific grant from any funding agency in the public, commercial, or not-for profit sectors. 
Fitzsimons, Grimes, Galvin.

\section{References}

1. Tam VC, Knowles SR, Cornish PL, Fine N, Marchesano R, Etchells EE. Frequency, type and clinical importance of medication history errors at admission to hospital: a systematic review. CMAJ 2005; 173: 510-5.

2. Institute for Healthcare Improvement (2006). Accuracy at Every Step: The Challenge of Medication Reconciliation. Cambridge, MA: Institute of Healthcare Improvement. http://www.ihi.org/lHI/Topics/PatientSafety/MedicationSystems/Changes/Reconcile+Medicatio ns+at+All+Transition+Points.htm (accessed 13 July 2010).

3. Bolas H, Brookes K, Scott M, McElnay J. Evaluation of a hospital-based community liaison pharmacy service in Northern Ireland. Pharm World Sci 2004; 26: 114-20.

4. Cornish PL, Knowles SR, Marchesano R, Tam V, Shadowitz S, Juurlink DN, Etchells EE. Unintended medication discrepancies at the time of hospital admission. Arch Intern Med 2005; 165: 424-9.

5. Pippins JR, Gandhi TK, Hamann C, Ndumele CD, Labonville SA, Diedrichsen EK, Carty MG, Karson AS, Bhan I, Coley CM, Liang CL, Turchin A, McCarthy PC, Schnipper JL. Classifying and predicting errors of inpatient medication reconciliation. $J$ Gen Intern Med 2008; 23: 1414-22.

6. Slee A, Farrar K, Hughes D, Constable S. Optimising medical treatment - how pharmacist-acquired medication histories have a positive impact on patient care. Pharm $J$ 2006; 277: 737-39.

7. Wong JD, Bajcar JM, Wong GG, Alibhai SMH, Huh J-H, Cesta A, Pond GR, Fernandes OA. Medication reconciliation at hospital discharge: evaluating discrepancies. Ann Pharmacother 2008; 42: 1373-9.

8. National Institute for Health and Clinical Excellence, National Patient Safety Agency. Technical patient safety solutions for medicines reconciliation on admission of adults to hospital. [Online]. 2007 December. [accessed 20 August 2008]. Available from:

www.nice.org.uk

9. Madden D. Building a culture of patient safety. Report of the Commission on Patient Safety and Quality Assurance. Dublin: Department of Health and Children, 2008.

10. de Winter S, Spriet I, Indevuyst C, Vanbrabant P, Desruelles D, Sabbe M, Gillet JB, Wilmer A, Willems L. Pharmacist- versus physician-acquired medication history: a prospective study at the emergency department. Qual Saf Health Care 2010.

11. Green CF, Burgul K, Armstrong DJ. A study of the use of medicine lists in medicines reconciliation: please remember this, a list is just a list. Int J Pharm Pract 2010; 18: 116-21.

12. Glintborg B, Hillestrom PR, Olsen LH, Dalhoff KP, Poulsen HE. Are patients reliable when self-reporting medication use? Validation of structured drug interviews and home visits by drug analysis and prescription data in acutely hospitalized patients. J Clin Pharmacol 2007; 47: 1440-9.

13. Karkov LL, Schytte-Hanssen S, Haugbolle LS. Discrepancies between sources providing the medication histories of acutely hospitalised patients. Pharm World Sci: 2010; 32 : 449-54.

14. Rees S, Thomas P, Shetty A, Makinde K. Drug history errors in the acute medical assessment unit quantified by use of the NPSA classification. Pharm J 2007; 279: 469-71.

15. Karapinar-Carkit F, Borgsteede S, Zoer J, Smit H, Egberts A, Van den Bemt PM.

Effect of medication reconciliation with and without patient counselling on the number of pharmaceutical interventions among patients discharged from the hospital. Ann Pharmacother 2009; 43: 1001-10.

16. Yach D. World Health Organisation. Adherence to long-term therapies: Evidence for action. Geneva: 2003.

17. Care Quality Commission (2009). National study. Managing patients' medicines after discharge from hospital. Care Quality Commission,

http://www.cac.org.uk/ db/ documents/Managing patients medicines after discharge from hospital.pdf .

18. Grimes T, Delaney T, Duggan C, Kelly JG, Graham IM. Survey of medication documentation at hospital discharge: implications for patient safety and continuity of care. Ir $J$ Med Sci 2008; 177: 93-7.

19. Witherington EMA, Pirzada OM, Avery AJ. Communication gaps and readmissions to hospital for patients aged 75 years and older: observational study. Quality and safety in healthcare 2008; 17: 71-75. 
20. Scullin C, Scott MG, Hogg A, McElnay JC. An innovative approach to integrated medicines management. J Eval Clin Pract 2007; 13: 781-8.

21. McFadzean E, Isles C, Moffat J, Norrie J, Stewart D. Is there a role for a prescribing pharmacist in preventing prescribing errors in a medical admission unit? Pharm J 2003; 270 : 896-99.

22. Lau HS, Florax C, Porsius AJ, De Boer A. The completeness of medication histories in hospital medical records of patients admitted to general internal medicine wards. $\mathrm{Br} \mathrm{J} \mathrm{Clin}$ Pharmacol 2000; 49: 597-603.

23. National Adult Literacy Agency. Facts and figures about literacy. Dublin. 16 March 2009 [Online pdf available from:www.nala.ie] (accessed 14 Sept 2009)

24. Grimes T, Fitzsimons M, Ni Shuilleabhain M, Kidd P, Ferrie T. Qualities of sources of pre-admission medication information: identifying relative weighting. Hospital Pharmacists' Association of Ireland Annual Educational Conference, Dublin, 2010.

25. Landis JR, Koch GG. The measurement of observer agreement for categorical data. Biometrics 1977; 33: 159-74.

26. Collins DJ, Nickless GD, Green CF. Medication histories: does anyone know what medicines a patient should be taking? Int J Pharm Pract 2004; 12: 173-78.

27. Foss S, Schmidt JR, Andersen T, Rasmussen JJ, Damsgaard J, Schaefer K, Munck LK. Congruence on medication between patients and physicians involved in patient course. Eur J Clin Pharmacol 2004; 59: 841-7.

28. Tulner LR, Kuper IM, Frankfort SV, van Campen JP, Koks CH, Brandjes DP, Beijnen $\mathrm{JH}$. Discrepancies in reported drug use in geriatric outpatients: relevance to adverse events and drug-drug interactions. Am J Geriatr Pharmacother 2009; 7: 93-104.

29. Vira T, Colquhoun M, Etchells E. Reconcilable differences: correcting medication errors at hospital admission and discharge. Qual Saf Health Care 2006; 15: $122-6$.

30. Britten N, Stevenson FA, Barry CA, Barber N, Bradley CP. Misunderstandings in prescribing decisions in general practice: qualitative study.[see comment]. BMJ 2000; 320: 484-8.

31. Stevenson FA, Barry CA, Britten N, Barber N, Brakley CP. Doctor-patient communication about drugs: evidence for shared decision making. Social Science \& Medicine 2000; 50: 829-49.

32. Nassaralla CL, Naessens JM, Chaudhry R, Hansen MA, Scheitel SM. Implementation of a medication reconciliation process in an ambulatory internal medicine clinic. Qual Saf Health Care 2007; 16: 90-4.

33. Alldred DP, Standage C, Zermansky AG, Barber ND, Raynor DK, Petty DR. The recording of drug sensitivities for older people living in care homes. Br J Clin Pharmacol 2010; 69: 553-57.

34. Farooq M, Kirke C, Foley K. Documentation of drug allergy on drug charts in patients presenting for surgery. Ir J Med Sci 2008; 177: 243-45.

35. Grimes T, Duggan C, Gallagher P, Strawbridge J. Care of the stroke patient communication between the community pharmacist and prescribers in the Republic of Ireland. Pharm World Sci 2009. Dec;31(6):648-55.

36. National Prescribing Centre. Medicines Reconciliation: A Guide to Implementation. Good practice guides, 5 minute guides, National Prescribing Centre, 2008.

37. Bedell SE, Jabbour S, Goldberg R, Glaser H, Gobble S, Young-Xu Y, Graboys TB, Ravid S. Discrepancies in the use of medications. Their extent and predictors in an outpatient practice. Arch Intern Med 2000; 160: 2129-34.

38. Grimes T. Potential hazards in the medication use process at the hospital community Interface and a strategy to reduce them. Dublin: Royal College of Surgeons in Ireland, 2009. 39. Cohen J. Statistical Power Analysis for the Behavioural Sciences $2^{\text {nd }}$ ed. Lawrence Erlbaum Associates; 1988. 


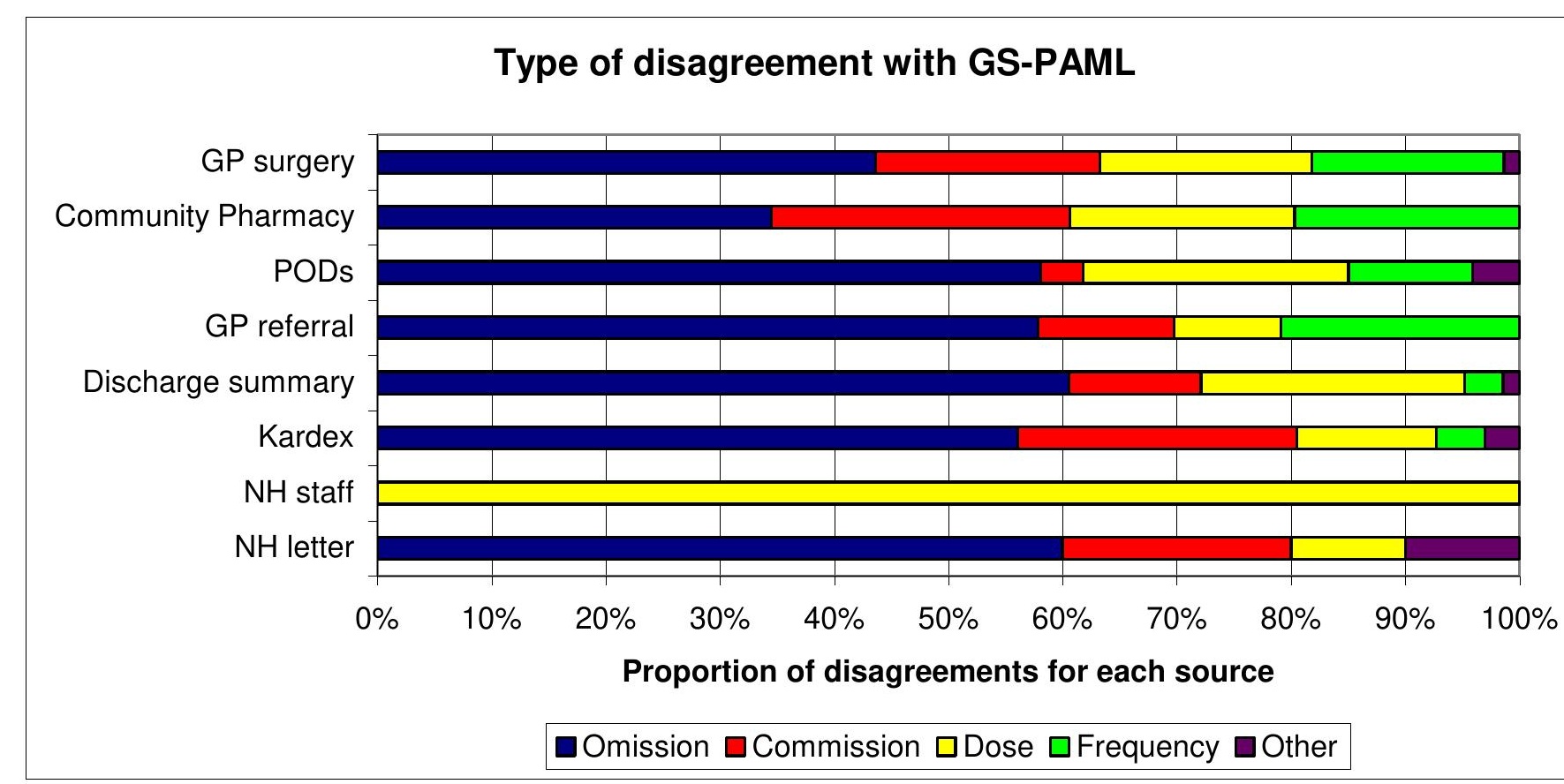

Figure 1. Types of disagreement with GS-PAML for each source

$\mathrm{GP}=$ General Practitioner

$\mathrm{POD}=$ Patient Own Drugs

$\mathrm{NH}=$ Nursing Home

GS-PAML = Gold Standard Pre-Admission Medication List 
Table 1. Performance of each PAM information source per patient $\mathbf{n}(\%)$

\begin{tabular}{|c|c|c|c|c|c|c|c|c|}
\hline Source & \begin{tabular}{|l|} 
A \\
Potential for \\
use \\
n (\%) \\
\end{tabular} & $\begin{array}{l}\text { B } \\
\text { Availability } \\
\text { n (\%) }\end{array}$ & \begin{tabular}{|l} 
C \\
Agreement with \\
GS-PAML per \\
patient \\
$\mathrm{n}(\%)$ \\
\end{tabular} & $\begin{array}{l}\text { Agreement with } \\
\text { GS-PAML per } \\
\text { medication } \\
\mathrm{n}(\%)\end{array}$ & $\begin{array}{l}\text { Disagreements } \\
\text { per patient } \\
\text { (mean) }\end{array}$ & $\begin{array}{l}\text { D } \\
\text { Allergy } \\
\text { agreement } \\
\mathrm{n}(\%) \\
\end{array}$ & $\begin{array}{l}\text { Grading } \\
\text { score } \\
A^{*}(B+C+D)\end{array}$ & Ranking \\
\hline Community pharmacy & $134(100)$ & $126(94)$ & $21(17)$ & $1025(77)$ & 2.5 & $89(71)$ & 182 & 1 \\
\hline GP surgery & $134(100)$ & $88(66)$ & $9(10)$ & $656(69)$ & 3.3 & $80(91)$ & 167 & 2 \\
\hline Inpatient Kardex & $50(37)$ & $34(68)$ & $2(6)$ & $262(62)$ & 4.8 & $26(77)$ & 56 & 3 \\
\hline POD & $134(100)$ & $58(43)$ & $2(3)$ & $350(57)$ & 4.6 & $\mathrm{~N} / \mathrm{A}$ & 46 & 4 \\
\hline GP referral letter & $53(40)$ & $42(79)$ & $1(2)$ & $189(44)$ & 6.2 & $10(24)$ & 42 & 5 \\
\hline Discharge summary & $50(37)$ & $29(58)$ & 1 (3) & $191(49)$ & 6.9 & $7(24)$ & 32 & 6 \\
\hline $\mathrm{NH}$ staff & $9(7)$ & $9(100)$ & $8(89)$ & $89(90)$ & 0.2 & $9(100)$ & 20 & 7 \\
\hline $\mathrm{NH}$ list & $9(7)$ & $6(67)$ & $1(17)$ & $125(98)$ & 1.7 & $4(67)$ & 11 & 8 \\
\hline
\end{tabular}

PAM = Pre-admission Medication

$\mathrm{GP}=$ General Practitioner

$\mathrm{POD}=$ Patient Own Drugs

$\mathrm{NH}=$ Nursing Home

GS-PAML = Gold Standard Pre-Admission Medication List 
Fitzsimons, Grimes, Galvin.

Table 2. Agreement between PAML and GS-PAML and use of one or more OTC medication.

\begin{tabular}{|l|l|l|l|l|l|}
\hline Source & $\begin{array}{l}\text { OTC medication not used } \\
\mathbf{n}(\%)\end{array}$ & $\begin{array}{l}\text { OTC medication used } \\
\mathbf{n}(\%)\end{array}$ & $\chi^{\mathbf{2}}$ & $\mathbf{d f}$ & $\mathbf{p}$ \\
\hline GP surgery & $9 / 62(15 \%)$ & $0 / 26(0 \%)$ & 4.2 & 1 & $<0.05$ \\
\hline Community pharmacy & $18 / 83(22 \%)$ & $3 / 43(7 \%)$ & 4.4 & 1 & $<0.05$ \\
\hline POD & $2 / 31(7 \%)$ & $0 / 27(0 \%)$ & 1.8 & 1 & NS \\
\hline GP referral letter & $1 / 33(3 \%)$ & $0 / 10(0 \%)$ & 0.3 & 1 & NS \\
\hline Inpatient Kardex & $2 / 17(12 \%)$ & $0 / 17(0 \%)$ & 2.1 & 1 & NS \\
\hline Discharge summary & $1 / 14(7 \%)$ & $0 / 15(0 \%)$ & 1.1 & 1 & NS \\
\hline
\end{tabular}

PAML $=$ Pre-Admission Medication List

GS-PAML $=$ Gold Standard Pre-Admission Medication List

OTC = Over The Counter

$\mathrm{GP}=$ General Practitioner

POD = Patient Own Drugs 
Fitzsimons, Grimes, Galvin.

Table 3. Correlation between number of drugs and number of disagreements on the PAML and the GS-PAML per list

\begin{tabular}{|l|l|l|l|}
\hline Source & Spearman's rho & Rho $^{2}$ & P value \\
\hline Community pharmacy & 0.431 & 18.6 & $\mathrm{p}<0.05$ \\
\hline GP surgery & 0.334 & 11.2 & $\mathrm{p}<0.05$ \\
\hline Inpatient Kardex & 0.468 & 21.9 & $\mathrm{p}<0.05$ \\
\hline POD & 0.327 & 10.7 & $\mathrm{p}<0.05$ \\
\hline GP referral letter & 0.559 & 31.2 & $\mathrm{p}<0.05$ \\
\hline Discharge summary & 0.472 & 22.3 & $\mathrm{p}<0.05$ \\
\hline $\mathrm{NH}$ staff & 0.134 & 1.81 & $\mathrm{NS}$ \\
\hline $\mathrm{NH}$ list & 0.140 & 1.96 & $\mathrm{NS}$ \\
\hline
\end{tabular}

Rho $^{2} 1-10 \%=$ weak correlation

$\mathrm{Rho}^{2} 10-25 \%=$ moderate correlation

$\mathrm{Rho}^{2}>25 \%=$ strong correlation [40]

$\mathrm{GP}=$ General Practitioner

POD = Patient Own Drugs

$\mathrm{NH}=$ Nursing Home

PAML = Pre-admission Medication List

GS-PAML = Gold Standard Pre-Admission Medication List 
Appendix 1. Medication history taking - patient interview questions approved by the National Adult Literacy Agency as Plain English

\section{Introduction}

My name is XXXX and I am one of the pharmacists here. I was hoping to have a quick chat with you about your medicines. One of my jobs is to make sure we have a full list of all the medications you were taking at home. So l'd like to know what medications you take, how much you take and how often you take them.

\section{General questions}

- Do you normally take medicines at home (including tablets, liquids, inhalers, patches, injections, creams, eye drops and so on)?

- What is (are) the name(s) of these medication(s)?

- Do you know how many different medicines you are taking (if person doesn't know medication names)?

- How strong is the medication (give an example of how you would measure strength)? How much of that medication do you take at a time?

- How many times a day do you take it?

- $\quad$ Do you have any medicines at home that you only take when you need them (instead of every day)?

\section{Over-the-counter and other non-prescription items}

- $\quad$ Do you take any medicines that are not prescribed for you by your doctor, for example over-the-counter medications that you can buy in a shop or pharmacy? These might include pain medications, stomach or bowel medications, vitamins, or herbal products.

- What is (are) the name(s) of these medication(s)?

- How strong is the medication (give an example of strength)? How much of that medication do you take at a time?

- How many times a day do you take it?

\section{Allergies and side effects}

- $\quad$ Have you ever had an allergic reaction to any medication? What happened?

- $\quad$ Have you ever had a side effect from any medicine? What happened?

\section{Ability to follow medication instructions}

- $\quad$ Do you always take your medicines as directed to by your doctor?

- $\quad$ Do you have any problems remembering to take your medicines at home?

- Do you ever miss doses?

\section{Community pharmacy and frequency of attendance}

- $\quad$ Do you tend to go to the same pharmacy most of the time?

- Would you say you always go to the same pharmacy, mostly go to the same one, sometimes go to it or rarely go to the same pharmacy more than once?

- If you do go to the same pharmacy, what is its name and where is it? 